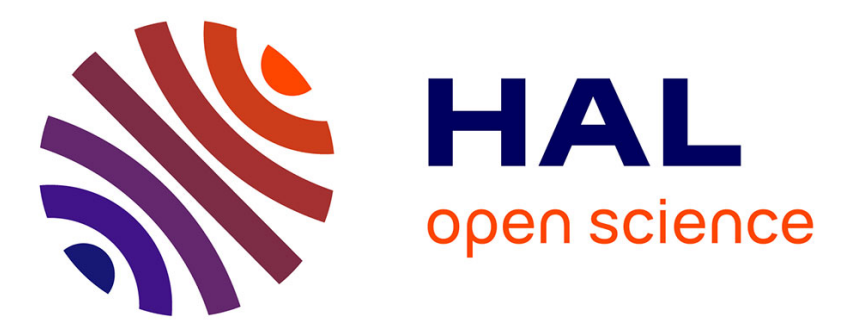

\title{
Dementia beyond 2025: knowledge and uncertainties
}

Paul-Ariel Kenigsberg, Jean-Pierre Aquino, Alain Berard, Fabrice Gzil, Sandrine Andrieu, Sube Banerjee, Francois Bremond, Luc Buee, Jiska Cohen-Mansfield, Francesca Mangialasche, et al.

\section{- To cite this version:}

Paul-Ariel Kenigsberg, Jean-Pierre Aquino, Alain Berard, Fabrice Gzil, Sandrine Andrieu, et al.. Dementia beyond 2025: knowledge and uncertainties. Dementia, 2015. hal-01850019

\section{HAL Id: hal-01850019 \\ https://hal.inria.fr/hal-01850019}

Submitted on 26 Jul 2018

HAL is a multi-disciplinary open access archive for the deposit and dissemination of scientific research documents, whether they are published or not. The documents may come from teaching and research institutions in France or abroad, or from public or private research centers.
L'archive ouverte pluridisciplinaire HAL, est destinée au dépôt et à la diffusion de documents scientifiques de niveau recherche, publiés ou non, émanant des établissements d'enseignement et de recherche français ou étrangers, des laboratoires publics ou privés. 


\section{Dementia beyond 2025: knowledge and uncertainties}

Paul-Ariel Kenigsberg, Jean-Pierre Aquino, Alain Bérard, Fabrice Gzil

Fondation Médéric Alzheimer, Paris, France

Sandrine Andrieu

INSERM UMR 1027, Université Paul-Sabatier, CHU Toulouse, Toulouse, France

\section{Sube Banerjee}

Brighton and Sussex Medical School, University of Sussex, Brighton, United Kingdom

\section{François Brémond}

INRIA, Sophia Antipolis, France

\section{Luc Buée}

INSERM UMR 837, CHR University of Lille, Lille, France

\section{Jiska Cohen-Mansfield}

Herczeg Institute on Aging, Tel-Aviv University, Israel

\section{Francesca Mangialasche}

Karolinska Institutet, Stockholm University, Stockholm, Sweden

\section{Hervé Platel}

INSERM U923, CHU Caen, France

\section{Eric Salmon}

CHU Liège and Cyclotron Research Centre, University of Liège, Belgium

\section{Philippe Robert}

CMRR Memory Center, CHU and CoBTeK, University of Nice Sophia Antipolis, France

Corresponding Author:

Paul-Ariel Kenigsberg, Fondation Médéric Alzheimer, 30 rue de Prony, 75017 Paris, France Email : kenigsberg@med-alz.org 


\section{Abstract}

Given that there may well be no significant advances in drug development before 2025, prevention of dementia/AD through the management of vascular and lifestyle-related risk factors may be a more realistic goal than treatment. Level of education and cognitive reserve assessment in neuropsychological testing deserve attention, as well as cultural, social and economic aspects of caregiving. Assistive technologies for dementia care remain complex. Serious games are emerging as virtual educational and pleasurable tools, designed for individual and cooperative skill-building. Public policies are likely to pursue improving awareness and understanding of dementia; providing good quality early diagnosis and intervention for all; improving quality of care from diagnosis to the end of life, using clinical and economic endpoints; delivering dementia strategies quicker, with an impact on more people. Dementia should remain presented as a stand-alone concept, distinct from frailty or loss of autonomy. The basic science of sensory impairment and social engagement in people with dementia needs to be developed. E-learning and serious games programmes may enhance public and professional education. Faced with funding shortage, new professional dynamics and economic models may emerge through coordinated, flexible research networks. Psychosocial research could be viewed as an investment in quality of care, rather than an academic achievement in a few centres of excellence. This would help provide a competitive advantage to the best operators. Stemming from care needs, a logical, systems approach to dementia care environment through organizational, architectural and psychosocial interventions may be developed, to help reduce symptoms in people with dementia and enhance quality of life. Dementia-friendly environments, culture and domesticity are key factors for such interventions.

Keywords: Dementia; Prospective ; Prevention; Psychosocial interventions; Care Environment 


\section{Introduction}

The Fondation Médéric Alzheimer initiated in 2007 a reflection on its future work on Alzheimer's disease (AD) and related disorders. The objective was to develop an original, up-to-date, robust and valid conceptual framework on how the future context of dementia may unfold, engaging with uncertainty within limits of scientific plausibility (Brodaty et al., 2011). The present article provides a synthesis of two international expert group meetings organized by the Fondation Médéric Alzheimer to delineate what is known, what is unknown and what may be plausible directions for the future. It is intended for a broad public: people with dementia and their families, dementia care professionals, policy-makers. This scientific outlook builds on experience gained from recent national policies in Europe. Furthermore, whenever possible, experts identified observable events of today that may become seeds of change for tomorrow. The future horizon has been set at 2025 .

\section{Disease scope}

$\mathrm{AD}$ and related diseases differ from neurologic pathologies affecting mostly motor and sensory functions, and from non-degenerative pathologies affecting the mental sphere. AD is clearly and significantly different from normal ageing: it is a pathological process, which can be observed in structural and metabolic brain imaging. However, neuroimaging can also show biomarkers of AD in cognitively healthy people. These cases are currently designated as asymptomatic stage of the disease; a pathological evolution may be observable if these people live long enough and do not compensate for the disease. At the individual level, it is very difficult to disentangle normal aging from the early cognitive impairment of dementia, especially in advanced age (85+ years). Frailty, for its part, refers to a state of vulnerability, reflecting multi-system physiological and cognitive changes, which may evolve into impaired abilities to perform daily life activities. Cognitive and functional frailty coexist, depending upon the capacities of the cognitively impaired persons and their living ecosystem. Therefore, a disability model, which considers impairments in body functions and structures, limitations in individual activity and restrictions in social participation, is useful (World Health Organization, 2002). It is important to discuss preserving the person's capacities rather than the sole biological aspects of the disease.

\section{Epidemiology, risk factors and prevention of dementia}

Prevalence of dementia will continue to rise worldwide as a consequence of demographic evolution, even if age-specific trend seems to be lower than previously expected (Larson, Yaffe \& Langa, 2013). There are no data for prevalence, incidence and life expectancy using the new criteria for AD definition (Norton, Matthews, Barnes, Yaffe \& Brayne, 2014). Natural history of this "extended" AD, partially studied through the control arms of clinical trials, is largely unknown. Many protective factors have been consistently found from large observational studies. Up to a third of AD cases worldwide are attributable to risk factors and could thus be prevented (Norton et al, 2014). Several epidemiology teams have shifted from observation to prevention intervention studies, based on the management of vascular and lifestyle-related risk factors. However, multiple individual and collective barriers to lifestyle change make prevention interventions very difficult. Data on efficacy of large ongoing preventive trials and adherence of elders in long-term prevention strategies are awaited to clarify whether reduction or removal of risk factors can substantially decrease AD or dementia incidence. Observable seeds for change include a large cooperation in the field of prevention across countries (European Dementia Prevention Initiative) (Mangialasche, 
Kivipelto, Solomon \& Fratiglioni, 2012). Useful measures at a State level would be to implement effective policies for individual and collective prevention, as well as policies towards agro-business companies and health insurance to change consumer behaviours such as those towards lipid-rich food (Gittelsohn \& Lee, 2013).

\section{Understanding of cognitive ageing and dementia}

Understanding the complexity of dementia causation remains a major endeavor. Dementia is not a simple disease, rather a syndrome (a set of signs and symptoms) with multiple interacting aetiologies (causes and factors), which can be clearly distinguished from normal ageing. Most preserved regions in normal aging are the hippocampus and posterior/cingulate regions (involved in strategic processes and episodic memory) which are part of the core process in AD pathology (Desgranges, Kalpouzos \& Eustache, 2008). AD is not a myth: it can be considered as a structural problem (specific atrophy with a decrease of synaptic connections in regions of the brain) coupled with a fall in cerebral metabolism. Vascular determinants of $\mathrm{AD}$ became a key issue, with new links between metabolic and vascular pathways being unveiled. The role of exercise, education and cognitive reserve (brain capacity to offset cognitive impairments) in the prevention of dementia will need further investigation. The role of different risk and protective factors can be clarified by ongoing epidemiological studies, using a life-course perspective to describe the complex and dynamic interactions between factors in determining age-associated neurodegeneration.

\section{Biological causes of $A D$ and dementia}

We remain almost entirely ignorant about the molecular mechanisms of AD and dementia. Since 2010, new insights emerged from molecular biology, but biological determinants of $\mathrm{AD}$ and dementia appear now more complex. Both tau and amyloid pathologies are relevant for $\mathrm{AD}$, but links between them are still ill-defined. Anatomically, tau-induced neurofibrillary degeneration is now well-described (Braak stages), with a very reproducible and specific sequence (Braak \& Del Tredici, 2012), as opposed to the distribution of amyloid burden. There is a huge overlap among neurodegenerative disorders, with common mechanisms such as a prion-like propagation and mixed pathologies (AD and vascular dementia). Better crosslinking of animal and human studies in neurodegenerative diseases should be encouraged. Although genome-wide association studies (GWAS) identified a number of new susceptibility genes in people with $\mathrm{AD}$, the exact role of these genes in complex biological regulation pathways remains elusive.

\section{Detection and diagnosis of dementia}

While the new Diagnostic and Statistical Manual of Mental Disorders (DSM-5) abandoned the word of dementia to propose major (and mild) neurocognitive disorders as new diagnostic categories, the concept of dementia remains widely used. Complete clinical neuropsychological assessment (comprehensive cognitive evaluation) remains today the only way to make an accurate diagnosis of early cognitive disorder in $\mathrm{AD}$ or other dementia. Cognitive testing is included in memory clinics and in research, but not sufficiently in professional practice in nursing homes. Underlying causes of memory disorders appear heterogeneous and sometimes difficult to determine, challenging predictions of individual disease course. In France, 25\% of diagnoses in memory centres remain pending (Le Duff et al, 2012). On another hand, neuroimaging techniques and research for diagnosis biomarkers developed rapidly in the past decade. Apart from cerebro-spinal fluid (CSF) amyloid, and 
positron emission tomography (PET) cerebral amyloid imaging, biomarkers reflect different pathological processes that may occur at different times of the disease evolution, with a variable importance between patients, and in different combinations. In patients with mild cognitive impairment, CSF amyloid is a predictive marker of $\mathrm{AD}$, but atrophy seen in magnetic resonance imaging (MRI) is a slightly better predictor of the rate of clinical decline. Task-free (resting) MRI and task-based MRI may prove useful as non-invasive biomarkers in studying the progression of memory failure over the course of AD. However, despite neuroimaging progress in biomarkers, their predictive power is not high enough to allow their use in screening. Blood biomarkers remain elusive. As a consequence, biomarkers are still reserved to research. It may still be so in the next ten years. Studies on biomarker validation will provide researchers with a better understanding of $\mathrm{AD}$ progression, in order to detect which systems are affected in its earliest stages. A better understanding of the underlying causes of dementia may eventually lead to more personalized therapeutic interventions, and in the development of new treatments that may modify the course of the disease. From the regulatory viewpoint, biomarker use became an important issue, to enrich the population recruited in randomized clinical trials in people with $\mathrm{AD}$ or other causes of cognitive decline. As early diagnosis may be of interest for earlier clinical trials, prognostic markers may eventually prove more useful than diagnostic ones. An early and timely diagnosis is important to facilitate adjustment of people and their family to living with the disease, and to adapt care modalities (Brooker, La Fontaine, Evans, Bray \& Saad, 2013).

\section{New therapeutic targets for AD}

A drug able to stop or reverse AD progression (i.e., disease-modifying drug) is not yet available. Between 2002 and 2012, 99.6\% of the 244 agents tested for efficacy in slowing the progression of $\mathrm{AD}$ failed to achieve their primary clinical endpoints (Ousset et al, 2014). The aetiology and pathophysiology of AD are not fully known, making it difficult to identify a proper target and develop an effective cure. We do not know when a disease-modifying drug for $\mathrm{AD}$ will be available. A different methodological approach is now being implemented to test anti-amyloid drugs, based on earlier intervention (at pre-symptomatic phase). This is done through the selection of subjects with genetic risk of familial AD or evidence of brain amyloid burden. New therapeutic approaches are being considered, including compounds regulating brain response to insulin and neuroinflammation. However, since AD is not a pure entity, better prevention appears more valuable than finding too many molecular targets for therapy.

\section{Psychosocial research and interventions in dementia}

More and more well conducted randomized studies show durable effect of psychosocial interventions (e.g. cognitive stimulation, music or art-therapy workshops)(Guétin et al, 2009), which have been shown to be effective in managing functional, behavioral and psychological symptoms of dementia. Basic processes involved in psychosocial interventions should be identified better (Cohen-Mansfield, Marx, Thein \& Dakheel-Ali, 2011). New methodological approaches associating quantitative and qualitative evaluation should be encouraged. Psychosocial research may explore whether psychosocial interventions, along or without psychiatric medication, enhance or not each other's impact. Cultural, social and economic aspects of caregiving deserve more attention. Protective factors, such as education and cognitive reserve, should be given more consideration in neuropsychology assessment and psychosocial interventions. Increased collaborative contracting is needed between neuropsychological research and clinical teams (particularly in nursing homes) in order to 
develop clinical trials together. Multicomponent non-pharmacological interventions, comprising information to caregivers or cognitive approach to facilitate daily activities of people with dementia, are effective, although their evaluation is complex (Brodaty \& Arasaratnam, 2012). Psychosocial intervention at home can be beneficial in reducing behavioral symptoms, when individually tailored and when applied during a sufficient period. Research is needed to understand the most efficient procedures for psychosocial intervention, their mechanisms of efficacy and the modulating factors. Early psychosocial intervention at home should be developed, based on an individual model of patients' needs. Stimulation approaches should be expanded to match the full range of cognitive and sensory impairments.

\section{Assistive technology and dementia}

Information and Communication Technologies (ICTs) can provide useful information for assisting older adults and also for assessing specific domains of AD patient's life (behaviour, cognition, functional status). Assistive technology must be specifically tailored to the enduser's capacities. Reliability of these ICTs is hard to achieve. Several initiatives have been organized to establish recommendations for ICT use in AD (Robert et al., 2013). Some clinical studies on long duration have shown the difficulties in identifying strong benefits of using ICTs for people with AD. More efforts in performance and evaluation of ICTs are needed to help industry meet user needs and general practitioners in prescribing the available technologies. A solid economic model is a major issue: who will pay for assistive technology? Who will install and maintain ICTs at AD patients' homes? The costeffectiveness balance for assistive technology remains a matter of debate. Serious games, adapted to people with dementia, may constitute an important tool to maintain autonomy (Robert et al., 2014). By providing pleasurable activities and person empowerment, these games are a way to enter the homes of people with dementia through technology, to structure collaborative care knowledge related to $\mathrm{AD}$ and to educate $\mathrm{AD}$ stakeholders cope with critical situations in patient everyday life. Establishing links between behavioural disorders and their causes could help a personal or virtual coach in proposing corrective actions and lifestyle training. The role of technology in improving sensory impairments, facilitating activities of daily living and providing pleasure is under-explored.

\section{Sensory impairment and dementia}

The relation between sensory impairment and dementia is under-studied. Sensory impairments may be either causal factors or consequences of dementia. Whether sensory impairments could be markers or modifiable risk factors for dementia remains debated. A double disability approach (dementia and sensory impairment) may bring new insight (McKeefry D. \& Bartlett R., 2010). Olfaction disorders as predictors of cognitive impairment remain a debated topic. Low vision is significantly associated to the development of dementia. People with a good or excellent vision may have a risk reduced by $37 \%$ to develop dementia within 8.5 years. People with a poorer vision who do not visit an ophthalmologist may have a 9.5-fold increased risk of $\mathrm{AD}$ and a 5-fold increased risk of cognitive impairment without dementia (Rogers \& Langa, 2010). Hearing loss is associated with an increased risk of incident dementia: 2-fold for mild, 3-fold for moderate and 5-fold for severe hearing loss (Lin et al., 2011). Non-corrected hearing loss with social discomfort is often confused with cognitive impairment, as people may fail to understand some high-pitch consonants or entire words in the questions of cognitive tests. People with dementia and delusions have also poorer vision and hearing. However, delusions may not represent psychotic symptoms: their true meaning may be related to disorientation, representation of reality, re-experience of past 
events, loneliness, insecurity, boredom, or other triggering events (Cohen-Mansfield, Golander, Ben-Israel \& Garfinkel, 2011). Delirium, an acute failure of the brain's ability to assimilate and respond appropriately to inner and outer stimuli, appears as a very strong risk factor for dementia in the oldest-old population (Davis et al, 2012), increasing dementia incidence by a factor of 9 , and dementia severity or global function by a factor of 3 . Proper identification of underlying causes, a suitable care environment and reorientation of the person may decrease adverse outcomes of delirium.

\section{Biomedical ethics and dementia}

A person-centred approach is essential for the well-being of people with dementia (Alzheimer Society of Canada, 2011). Challenging the stigma associated with dementia and promoting initiatives enabling people with dementia and their families to live well in the community is also of great importance (Van Gorp \& Vercruysse, 2011). Assessment of decision-making capacity (e.g. to consent to treatment or research, to appoint a proxy, to vote) has emerged as a new area of practice and research, with a shift from a diagnosis-based approach to the consideration of key functional abilities. New criteria and tools for AD diagnosis create an opportunity for an earlier diagnosis, which holds great interest for clinical research but which may be ethically sensitive if translated in clinical practice. Considering AD and dementia through the framework of the disability model creates an opportunity to understand better the importance of psychosocial interventions and of societal changes aimed at social participation. Some ethical issues have not yet received as much attention as one might have expected: research in nursing homes, clinical trials for complex cases and effects of cultural differences on attitudes and practices about dementia. The ethics of assistive technology has been discussed in general terms, but a framework to analyze the ethical issues related to the use of a particular technology in a given context is still missing. Communication about AD research (funding and results) deserves more care, as it can reinforce stigma and give unrealistic hopes (Gzil, 2013).

\section{Care for people with dementia}

Dementia care involves multiple domains: functional, cognitive and intellectual, emotional, medical (including pain management), and end-of-life care. Behavioural and psychological symptoms of dementia (BPSD) are extremely important in explaining the burden of the disease for the persons themselves, caregivers and society. BPSD relate to unmet needs of people with dementia, mostly pain and discomfort, need of social contact and support, and need of stimulation against boredom (Cohen-Mansfield, 2013a). There is evidence that agitated behavior responds to pain management (Husebo, Ballard, Cohen-Mansfield, Seifert $\&$ Aarsland, 2014). Although non-pharmacological interventions are recommended as firstline therapy for BPSD, a number of barriers to their delivery have been observed, which may be related to the people with dementia, staff, families, environment or systems processes (Cohen-Mansfield, Thein \& Marx, 2014). Stemming from care needs, a logical, systems approach of the care environment through organizational, architectural and psychosocial interventions needs therefore to be developed. Future of psychosocial interventions may include the notion of "successful dementia" (Cohen-Mansfield et al, 2013b) to prevent negative affect and agitation while maximizing indications of positive affect, such as contentment and pleasure (Cohen-Mansfield, 2011). This involves a shared understanding of non-pharmacological intervention on communication and teamwork in care services; enhancing a multidisciplinary approach that would allow for the tailoring and individualization that is required of successful interventions; the integration of clinical 
research and systems (organizational) research; research in nursing homes and research in individual homes. Dementia-friendly environments, culture (collective habits, values and beliefs acquired in human societies) and domesticity are key factors for such interventions. A new dynamics of research might evolve through coordinated, flexible research networks, involving field and university professionals; research in dementia care could become a marker of quality for innovative nursing homes.

\section{Public policies, economics and dementia}

Early diagnosis and early intervention should be a priority in public policy, to help organize the care pathway, to enable people with dementia and their families to make their own choices and to prevent the harms that accrue from not knowing what is going on. Policy focuses on spending now to save in the long term. Diagnosis and treatment became a right. Direct measurements of cost and effectiveness of interventions are scarce. Programmes, strategies and initiatives must be evaluated on a qualitative and quantitative basis, to assess reproducibility in different settings. The best models of care are poorly diffused and implemented. The number of countries having implemented a National Dementia Strategy is expanding gradually. Recently, WHO and the G8 countries have given dementia a status of public health priority, raising policy attention worldwide. Dementia is very expensive, with high costs stemming from the cost of care. Many services, such as care homes, offer poor value for money. There is a need for a better understanding of the cost drivers, as well as dementia and ageing as a macro-economic issue. We have no comprehensive models for dementia costs over time. The cost of inaction (not doing anything) should be integrated into the costing model, to remove perverse incentives. Further developments in the economics of dementia may increase political will and focus. Public policies are likely to pursue improving awareness and understanding of dementia; providing good quality early diagnosis and intervention for all; improving quality of care from diagnosis to the end of life, using clinical and economic endpoints; delivering dementia strategies quicker, with an impact on more people.

\section{Funding}

This work has been initiated and supported solely by Fondation Médéric Alzheimer (France), a State-approved, public interest, non-profit foundation dedicated to Alzheimer's Disease. Since 1999, its primary objective has been to promote social and psychosocial care initiatives aimed at improving the quality of life of caregivers of people with dementia. It has no interest or other involvement in Alzheimer's Disease treatment development. 


\section{References}

Alzheimer Society of Canada (2011). Guidelines for Care. Person-centred care of people with dementia living in care homes. Framework. Toronto: Alzheimer Society of Canada.

Braak, H. and Del Tredici, K. (2012). Where, when, and in what form does sporadic Alzheimer's disease begin? Curr Opin Neurol, 25, 708-714.

Brodaty, H., Breteler M. M., DeKosky, S. T., Dorenlot, P., Fratiglioni, L., Hock, C., Kenigsberg, P. A., Scheltens, P. \& De Strooper, B. (2011). The world of dementia beyond 2020. J Am Geriatr Soc, 59, 923-927.

Brodaty, H. \& Arasaratnam, C. (2012). Meta-analysis of nonpharmacological interventions for neuropsychiatric symptoms of dementia. Am J Psychiatry, 169(9), 946-953.

Brooker, D., La Fontaine, J., Evans, S., Bray, J. \& Saad, K. (2014). Alzheimer Cooperative Valuation in Europe. Timely diagnosis of dementia. 2013 synthesis report. Int J Geriatr Psychiatr 29(7), 682-693.

Cohen-Mansfield, J. (2011). The shifting baseline theory of well-being: lessons from across the aging spectrum. In: Poon, L.W., Cohen-Mansfield, J., eds. Understanding well-being in the oldest old. New York: Cambridge University Press, pp 46-60.

Cohen-Mansfield, J., Marx, M. S., Thein, K. \& Dakheel-Ali, M. (2011). The impact of stimuli on affect in persons with dementia. J Clin Psychiatry, 72, 480-486.

Cohen-Mansfield, J., Golander, H., Ben-Israel, J. \& Garfinkel, D. (2011). The meanings of delusions in dementia; a preliminary study. Psychiatry Res, 189, 97-104.

Cohen-Mansfield, J. (2013a). Nonpharmacologic treatment of behavioral disorders in dementia. Curr Treat Options Neurol, 15(6),765-785.

Cohen-Mansfield, J. (2013b). Advances in Alzheimer's Research: Implications for Family Caregiving. In: Zarit, S. H., Talley, R. C., eds. Caregiving for Alzheimer's Disease and Related Disorders. New York: Springer, pp 181-202.

Cohen-Mansfield, J, Thein, K. \& Marx, M. S. (2014) Predictors of the impact of nonpharmacologic interventions for agitation in nursing home residents with advanced dementia. J Clin Psychiatry, 75(7), e666-671.

Davis, D. H. J., Muniz Terrera, G., Keage, H., Rahkonen, T., Oinas, M., Matthews, F. E., Cunningham, C., Polvikoski, T., Sulkava, R., MacLullich, A. M. \& Brayne, C. (2012). Delirium is a strong risk factor for dementia in the oldest-old: a population-based cohort study. Brain, 135, 2809-2816.

Desgranges, B., Kalpouzos, G. \& Eustache, F. (2008). Cerebral imaging in healthy aging: contrast with Alzheimer disease. Rev Neurol (Paris), 164, S102-7.

Dubois, B., Feldman, H. H,, Jacova, C., Hampel, H., Molinuevo, J. L., Blennow, K., DeKosky, S. T., Gauthier, S., Selkoe, D., Bateman, R., Cappa, S., Crutch, S., Engelborghs, S., Frisoni, G. B., Fox, N. C., Galasko, D., Habert, M. O,, Jicha, G. A., Nordberg, A., Pasquier, F., Rabinovici, G., Robert, P., Rowe, C., Salloway, S., Sarazin, M., Epelbaum, S., de Souza, L.C., Vellas, B., Visser, P.J., Schneider, L., 
Stern, Y., Scheltens, P. \& Cummings, J. L. (2014). Advancing research diagnostic criteria for Alzheimer's disease: the IWG-2 criteria. Lancet Neurol, 13(6), 614-629.

Gittelsohn, J. \& Lee, K. (2013). Integrating Educational, Environmental, and Behavioral Economic Strategies May Improve the Effectiveness of Obesity Interventions. Appl Econ Perspect Policy, 35, 52-68.

Guétin, S., Portet, F., Picot, M. C., Pommié, C., Messaoudi, M., Djabelkir, L., Olsen, A. L., Cano, M. M., Lecourt, E. \& Touchon J. (2009). Effect of music therapy on anxiety and depression in patients with Alzheimer's type dementia: randomised, controlled study. Dement Geriatr Cogn Disord, 28, 36-46.

Gzil F. (2013). Enjeux éthiques de la recherche sur la maladie d'Alzheimer. In : Tillement, J. P., Hauw, J. J., Papadopoulos, V. (eds). Le défi de la maladie d'Alzheimer, Synergies franco-québécoises. Académie nationale de médecine. Paris : Lavoisier, 2013, pp 25-31.

Husebo, B. S., Ballard, C., Cohen-Mansfield, J., Seifert, R. \& Aarsland, D. (2014). The Response of Agitated Behavior to Pain Management in Persons with Dementia. Am J Geriatr Psychiatry, 22(7), 708-717.

Larson, E. B., Yaffe, K. \& Langa K. M. (2013). New insights into the dementia epidemic. N Engl J Med 2013, 369(24), 2275-2257.

Le Duff, F., Develay, A. E., Quetel, J., Lafay, P., Schück, S., Pradier, C., Robert, P. \& the French National Alzheimer Databank (BNA) (2012). The 2008-2012 French Alzheimer plan: description of the national Alzheimer information system. $J$ Alzheimers Dis, 29, 891-902.

Lin, F. R., Metter, E. J., O’Brien, R. J., Resnick, S. M., Zonderman, A. B. \& Ferrucci, L. (2011). Hearing Loss and Incident Dementia. Arch Neurol, 68, 214-220.

Mangialasche, F., Kivipelto, M., Solomon, A. \& Fratiglioni, L. (2012) Dementia prevention: current epidemiological evidence and future perspective. Alz Res Ther, 4(1), 6.

Norton, S., Matthews, F. E., Barnes, D. E., Yaffe, K. \& Brayne C. (2014). Potential for primary prevention of Alzheimer's disease: an analysis of population-based data. Lancet Neurol, 13(8), 788-794.

Ousset, P. J., Cummings, J., Delrieu, J., Legrand, V., Prins, N., Winblad, B., Touchon, J., Weiner, M. W. \& Vellas, B. (2014). Is Alzheimer's disease drug development broken? What must be improved? J Prev Alz Dis, 1(1), 40-45.

Robert, P. H, König, A., Amieva, H., Andrieu, S., Bremond, F., Bullock, R., Ceccaldi, M., Dubois, B., Gauthier, S., Kenigsberg, P. A., Nave, S., Orgogozo, J. M., Piano, J., Benoit, M., Touchon, J., Vellas, B., Yesavage, J. \& Manera, V. (2014). Recommendations for the use of Serious Games in people with Alzheimer's Disease, related disorders and frailty. Front Aging Neurosci, 6, 54.

Robert, P. H., König, A., Andrieu, S., Bremond, F., Chemin, I., Chung, P. C., Dartigues, J. F., Dubois, B., Feutren, G., Guillemaud, R., Kenigsberg, P. A., Nave, S., Vellas, B., Verhey, F., Yesavage, J. \& Mallea, P. (2013). Recommendations for ICT use in Alzheimer's Disease. Monaco CTAD expert meeting. J Nutr Health Aging, 17, 653660.

Rogers, M. A. \& Langa, K.M. (2010). Untreated poor vision: a contributing factor to latelife dementia. Am J Epidemiol, 171, 728-735. 
McKeefry D. \& Bartlett R. (2010). Improving vision and eye health care to people with dementia. Research discussion paper 8. London: Thomas Pocklington Trust.

Van Gorp B. \& Vercruysse T. (2011). Framing and re-framing: A different way of communicating about Alzheimer's disease. Brussels: King Baudoin Foundation 2011.

World Health Organization (2002). International Classification of Functioning, Disability and Health: ICF. Geneva: World Health Organization.

Paul-Ariel Kenigsberg, $\mathrm{PhD}$, MBA, is Deputy Manager of the Coordination, Prospective and Strategy unit at the Fondation Médéric Alzheimer. He is chief editor of the national and international Press Review of the Fondation. This knowledge base, unique in its kind, established more than ten years ago, allows prospective watch-keeping of the context of $\mathrm{AD}$ in its scientific, medical, technological, social, cultural, professional, political, economic, legal aspects, and spotting of innovative initiatives worldwide. $\mathrm{He}$ also leads the prospective expert groups of the Fondation Médéric Alzheimer. His current work focuses on the sensory approach in Alzheimer's disease and on technologies for people with dementia and their caregivers.

Jean-Pierre Aquino, MD, specialized in Geriatrics and Public Health. Head Physician of the Porte Verte Medical Clinic in Versailles, Technical Advisor of the Fondation Médéric Alzheimer, he has been president of the French Society of Geriatrics and Gerontology (SFGG). Under these different titles, he participated in the Ménard Commission's work and in several working parties of the French Alzheimer Plan 2008-2012. As a President of the Committee on Advancing in age, prevention and quality of life, he provided a report to the French government titled "Anticipate for a preserved autonomy: a societal issue", used to prepare the upcoming Law for adapting the Society to Ageing.

Alain Bérard, MD, specialized in Public Health, economist and jurist, is Deputy Director of the Fondation Médéric Alzheimer where he also manages the Coordination, Prospective and Strategy unit. His research work and publications focus on the needs of people of 65 years old and above, on the different professions working with people with dementia, on the social and asset management behaviors of people with young-onset dementia, and more recently on sensory impairments and Alzheimer's disease. He also performs evaluation of health, social and medico-social services for modelling and dissemination purposes.

Fabrice Gzil is Doctor in Philosophy and Manager of the Studies Department at the Fondation Médéric Alzheimer. In his doctoral thesis, he studied the history of the discovery of Alzheimer's disease, animal models of cognitive impairments related to ageing and moral philosophy issues raised by the progressive alteration of discernment and judgment. At the Fondation Médéric Alzheimer, he is in charge of providing support to psychosocial research and research in human and social sciences. His current work focuses on care ethics and research in the context of dementia, as well as on the ways to promote rights, freedom and citizenship of people with cognitive disabilities. 
Sandrine Andrieu, MD, $\mathrm{PhD}$ in Aging and Public Health, is Professor of Epidemiology and Public Health at the Toulouse University school of Medicine. Since 2011, she has been leading the INSERM - Toulouse University research Unit UMR1027 on "Risks, chronic diseases and disability" and the "Aging and Alzheimer Disease" research team. She coordinates the French Observatory of Research in Alzheimer's Disease. As manager of the methodology clinical research unit of the Toulouse University hospital, she is in charge of methodological aspects of trials in aging. She is involved in large intervention studies in the field of neurodegenerative disease (PLASA Study, GuidAge Study, MAPT Study) and large cohorts of Alzheimer Disease patients (ICTUS, REAL.fr).

Sube Banerjee, MD, MBA, is Professor of Dementia, Associate Dean for Strategy and Director of Centre for Dementia Studies at the Brighton and Sussex Medical School. He developed the National Dementia Strategy for England. His current research focuses on the improvement of the quality of life and care of older people with mental health problems, particularly dementia.

François Brémond, $\mathrm{PhD}$, is Research Director at Inria and he is leading the STARS team at INRIA Sophia Antipolis, France. He designs and develops generic systems for dynamic scene interpretation. The targeted class of applications is the automatic interpretation of indoor and outdoor scenes observed with various sensors and in particular with static video cameras. These systems detect and track mobile objects, which can be either humans or vehicles, and recognize their behaviours. $\mathrm{He}$ is particularly interested in filling the gap between sensor information (pixel level) and recognized activities (semantic level). He is a co-founder of the Nice University CoBTeK team dedicated to the studies of ICT technologies for a better understanding of behavioural disorders.

Luc Buée, $\mathrm{PhD}$, is Director of the Alzheimer \& Tauopathies research team, INSERM UMR 837, at the Lille University Hospital. His research focuses on Alzheimer's disease and a group of neurodegenerative diseases characterized by an intracellular aggregation of microtubule-associated tau proteins. His teams develops experimental models for a better understanding of pathophysiological mechanisms underlying cognitive disorders, using molecular, biochemical, electrophysiological and behavioural techniques.

Jiska Cohen-Mansfield, $\mathrm{PhD}$ in Clinical Psychology and MBA, is Professor at the Department of Health Promotion at the School of Public Health and the director of Minerva Center for the Interdisciplinary Study of End of Life at Tel-Aviv University, Israel. Her work focuses on health and mental health promotion of older persons, including decreasing loneliness in older community dwelling persons, improving quality of life for persons with dementia by understanding the perspective of the person with dementia, and increasing motivation for physical activity among older persons. She is also interested in environmental design and technological innovations that can improve wellbeing of older persons, and in exploring avenues for improving the end of life experience of older persons. 
Francesca Mangialasche MD, specialized in Geriatrics, with a PhD in Medical Science. Her research activity is based on a scientific collaboration between the Aging Research Center, Karolinska Institutet/Stockholm University, Stockholm, Sweden; the Institute of Gerontology and Geriatrics, University of Perugia, Italy and the Department of Neurology, University of Eastern Finland (Kuopio campus). Her research focuses on clinical and neuropsychological aspects of brain aging and dementia, and on biological studies of brain aging.

Hervé Platel is Professor of Neuropsychology at the Caen University (INSERM unit U1077). In the 1990s, he was one of the first research workers to use neuro-imaging techniques to visualize brain activity during the analysis of the different perceptive components of music. His work enable the use of music as original material to study cognitive functions and as psychosocial intervention in dementia care.

Eric Salmon MD, $\mathrm{PhD}$, Neurologist, Professor, is Head of the Memory Centre, Department of Neurology at the University Hospital of Liège, Belgium; Medical Director of the Cyclotron Research Centre, University of Liège; Principal Investigator in the EC projects NEST-DD, European Alzheimer Disease Consortium (5th FW) and DiMI (6th FW), in Belgian InterUniversity Attraction Poles, in several clinico-pharmacological studies. Teaching in the Faculties of Medicine and of Psychology, University of Liège, he is involved in InterUniversity teaching programs, and in national and international research programs on neurodegenerative diseases, cognitive functions and functional imaging.

Philippe Robert is Professor of Psychiatry at the Nice School of Medicine, Director of the Nice Memory Centre for Care and Research, Director of the Cognition, Behaviour \& Technology Unit (CoBTeK) at the Nice Sophia Antipolis University. $\mathrm{He}$ is the coordinator of the French National Alzheimer data bank. The CoBTeK unit aims to develop and transfer new technologies, developed for the benefit of clinical medicine and most particularly elderly subjects and others patients suffering from neuropsychiatric / neurodevelopmental disorders. CoBTeK provide an optimal partnership between engineers (INRIA) and clinicians (Memory center, CMRR) corresponding to the "ICT or computer-to-clinic" link. 
Table 1: What is known?

Field

Disease scope

Epidemiology, risk factors and prevention

Understanding of cognitive ageing and dementia

Biological causes of dementia

Detection and diagnosis of dementia

New therapeutic targets for dementia

Psychosocial research and interventions in dementia

Assistive technology and dementia

Sensory impairment and dementia

\section{Current knowledge}

$\mathrm{AD}$ and related diseases differ from healthy ageing, from neurologic pathologies affecting mostly motor and sensory functions, and from non-degenerative pathologies affecting the mental sphere.

Prevalence of dementia will continue to rise worldwide as a consequence of demographic evolution even if agespecific trend seems to be lower than previously expected.

Dementia is a syndrome with multiple interacting causes, which can be clearly distinguished from healthy ageing. $\mathrm{AD}$ can be considered as a structural problem (specific atrophy with a decrease in synaptic connections in regions of the brain) coupled with a fall in cerebral metabolism.

Biological determinants of AD and dementia are complex. The anatomic sequence of neurofibrillary degeneration (tau pathology) is specific and reproducible, as opposed to the distribution of the amyloid burden.

Complete clinical neuropsychological assessment (comprehensive cognitive evaluation) is today the only way to make an accurate diagnosis of early neurocognitive disorder in $\mathrm{AD}$ or related diseases. Biomarkers remain reserved to research.

A drug able to stop or reverse $\mathrm{AD}$ progression is not yet available.

Multi-component psychosocial interventions are effective in managing functional, behavioral and psychological symptoms of dementia.

ICTs can provide useful information for assisting older adults and also for assessing specific domains of $\mathrm{AD}$ patient's life (behaviour, cognition, activities of daily living). Assistive technology must be specifically tailored to the end-user's capacities.

There is some evidence for an association (not a causality) between sensory impairment and dementia. Non-corrected vision and hearing loss are associated with an increased 
Biomedical ethics and dementia

Dementia care

Public policies, economics and dementia risk of incident dementia. Delirium, brain's failure to assimilate and respond appropriately to inner and outer stimuli, is a very strong risk factor for dementia in the oldest-old.

A person-centred approach is essential for the well-being of people with dementia. The disability framework model creates an opportunity to understand better the importance of psychosocial interventions and of societal changes aimed at social participation.

Behavioural and psychological symptoms of dementia (BPSD), major determinants of caregiver burden, relate to unmet needs of people with dementia, mostly pain and discomfort, need of social contact and support, and need of stimulation against boredom. Non-pharmacological interventions are recommended as first-line therapy for BPSD. Agitated behavior responds to pain management.

Early diagnosis and early interventions are priorities. Policy focuses on spending now to save in the long term. Diagnosis and treatment became a right. Dementia is very expensive, with high costs stemming from the cost of care. Many services, such as care homes, offer poor value for money. 


\section{Table 2: What is unknown?}

\section{Field}

Disease scope

Epidemiology, risk factors and prevention

Understanding of cognitive ageing and dementia

Biological causes of dementia

Detection and diagnosis

New therapeutic targets

Psychosocial research and interventions in dementia

Assistive technology in dementia

\section{Current uncertainties}

The individual course of dementia is hardly predictable. Cognitive and functional frailty coexist, depending upon the capacities of the impaired persons and their living ecosystem.

There are no data for prevalence, incidence and life expectancy using the new criteria for AD definition. Data on efficacy of large ongoing preventive trials and adherence of elders in long-term prevention strategies are awaited. Interventions based on the management of vascular and lifestyle-related risk factors may help prevent up to a third of AD cases worldwide.

The role of different risk and protective factors can be clarified using a life-course perspective to describe the complex and dynamic interactions between factors in determining age-associated neurodegeneration.

Molecular mechanisms of AD and dementia are still largely unknown. Links between amyloid and tau pathologies are still ill-defined. The exact role of susceptibility genes in complex biological regulation pathways remains elusive.

There are no simple tests for AD or dementia. The predictive value of biomarkers is not high enough to allow their use in screening. It may remain so in the next ten years.

The aetiology and pathophysiology of AD are not fully known, making it difficult to identify proper targets and develop an effective cure. We do not know when a diseasemodifying drug for $\mathrm{AD}$ will be available.

Research is needed to understand the most efficient procedures for psychosocial intervention, the mechanisms of efficacy of the interventions and the modulating factors. Cultural, social and economic aspects of caregiving deserve more attention.

More efforts in performance and evaluation of ICTs are needed to help industry meet user needs and general practitioners in prescribing the available technologies. A 
Dementia and sensory impairment

Biomedical ethics and dementia

Dementia care

Public policies, economics and dementia solid economic model is a major issue for assistive technology development in dementia.

Sensory impairments may be either causal factors or consequences of dementia. Whether sensory impairments could be markers or modifiable risk factors for dementia remains debated.

Research in nursing homes, clinical trials for complex cases, context analysis in technology implementation and effects of cultural differences on attitudes and practices about dementia deserve more attention.

The care system itself may produce adverse events in dementia care. Stemming from care needs, a logical, systems approach of the care environment through organizational, architectural and psychosocial interventions needs to be developed.

Direct measurements of cost and effectiveness of interventions are scarce. The best models of care are poorly diffused and implemented. There are no comprehensive models for dementia costs over time. 


\section{Table 3: What are plausible directions for the future?}

\section{Field}

Disease scope

Epidemiology, risk factors and prevention

Understanding of cognitive ageing and dementia

Biological causes of dementia

Detection and diagnosis of dementia

New therapeutic targets

Psychosocial research and interventions in dementia
Plausible directions for the future

It is important to discuss preserving the capacities of the cognitively impaired persons in their living ecosystem, rather than the sole biological aspects of the disease. A disability model, considering impairments in body functions and structures, limitations in individual activity and restrictions in social participations, is useful.

A large cooperative prevention initiative has been set up in Europe. Effective policies at a State level may include individual and collective prevention, as well as policies towards agro-business companies and health insurance to change consumer behaviours such as those towards lipid-rich food. Better prevention may be more valuable than finding too many therapeutic targets, since $\mathrm{AD}$ is not a pure entity.

Studies on biomarker validation will provide researchers with a better understanding of $\mathrm{AD}$ progression in its earliest stages.

There is a huge overlap among neurodegenerative disorders, with some common mechanisms. Better cross-linking of animal and human studies in neurodegenerative diseases should be encouraged.

An early diagnosis may be of interest for earlier clinical trials. Prognostic markers, rather than diagnostic ones, may prove more useful. Task-free MRI and task-based MRI may prove useful as non-invasive biomarkers in studying the progression of memory failure over the course of AD.

Drugs are being tested at the pre-symptomatic phase, through the selection of subjects with genetic risk of familial AD or evidence of brain amyloid burden. New therapeutic approaches include compounds regulating brain response to insulin and neuroinflammation.

New methodological approaches associating quantitative and qualitative evaluation should be 
Assistive technology and dementia

Dementia and sensory impairment

Biomedical ethics and dementia

Dementia care

Public policies, economics and dementia encouraged, as well as research in nursing homes. Early psychosocial intervention at home should be developed, based on an individual model of patients' needs, support and modulating factors. Stimulation approaches should be expanded to match the full range of cognitive impairments and sensory impairments.

Serious games, with the notion of pleasurable activities and person empowerment, may structure collaborative care knowledge related to $\mathrm{AD}$ and educate $\mathrm{AD}$ stakeholders cope with critical situations in patient everyday life.

Collaborative research between associations active in sensory impairment and those active in dementia care, may bring new insight, with a double disability approach. Correcting vision and hearing loss may help reduce cognitive impairment. Reorientation and environmental stimulation may reduce delirium in the hospitalized elderly.

Communication about AD research (funding and results) deserves more care, as it can reinforce stigma and give unrealistic hopes.

"Successful dementia" involves a shared understanding of non-pharmacological intervention in care services. Tailoring, dementia-friendly environments, culture and domesticity are key factors for such interventions. Research in dementia care, through coordinated, flexible research networks, involving field and university professionals, could become a marker of quality for innovative nursing homes.

The number of countries having implemented a National Dementia Strategy is expanding. WHO recognition of dementia as public health priority raises worldwide attention. Further developments in economics of dementia may increase political will and focus. 